\title{
Reducing inappropriate arterial blood gas testing in a level III intensive care unit: a before-and-after observational study
}

\author{
Oliver M Walsh, Katelyn Davis and Jonathan Gatward \\ www.doi.org/10.51893/2020.4.oa10 \\ Published online first 7 December 2020
}

Efforts to curtail unnecessary testing in medicine are growing. This has been demonstrated by the Choosing Wisely campaign, which has support from more than 100 medical societies across 20 countries, ${ }^{1}$ including the Australian and New Zealand College of Intensive Care Medicine $^{2}$ and the Society of Critical Care Medicine. ${ }^{3}$ Pathology tests are costly and contribute to the development of anaemia, which affects $95 \%$ of intensive care patients by Day 3 of admission. ${ }^{4}$ Almost half of all critical care patients receive red blood cell transfusions during their stay, ${ }^{5,6}$ with up to one-third of all transfusions attributed to this socalled anaemia of chronic investigation. ${ }^{7-9}$

Arterial blood gas ( $A B G$ ) analysis is the most commonly ordered test in the intensive care unit (ICU), ${ }^{10,11}$ with reports estimating that sampling is done, on average, 4.88.5 times per patient per day. ${ }^{10-18}$ No validated guidelines are available for $A B G$ sampling ${ }^{19}$ and ordering patterns appear to be primarily driven by cultural factors. ${ }^{14,15,20,21}$ Data suggest that $A B G$ tests are commonly performed for routine surveillance. One group of researchers found that more than $60 \%$ of all $A B G$ tests were performed at regular intervals for monitoring, after changes in ventilator settings, and before and after extubation, while only $26 \%$ were ordered in response to a respiratory event. ${ }^{20}$ A survey of critical care clinicians in a tertiary 98-bed ICU in the United States found that $90 \%$ of clinicians ordered $A B G$ tests for routine surveillance, $80 \%$ after every ventilation adjustment, $70 \%$ during spontaneous breathing trials before extubation and $65 \%$ for "convenience" when an arterial catheter was in situ. ${ }^{19}$ The presence of an arterial line has been shown to be the most powerful predictor of the number of $A B G$ tests ordered when controlled for illness severity. ${ }^{16,22,23}$ Ultimately, it has been suggested that $33-66 \%$ of all $A B G$ tests may not be clinically justified. ${ }^{14,15,17,19,20}$

Staff education with or without implementing a clinical guideline has been shown to decrease the number of $A B G$ tests performed in ICUs by $25-51 \%$, without obvious detrimental effects on patient care. 13-15,17-19 This effect is even greater in intubated patients ${ }^{13}$ and has been sustained for up to 5 years. ${ }^{24}$ Therefore, the aim of this study was to

\begin{abstract}
Background: Arterial blood gas ( $A B G$ ) analysis is the most frequently performed test in intensive care units (ICUs), often without a specific clinical indication. This is costly and contributes to iatrogenic anaemia.

Objectives: To reduce the number of ABG tests performed and the proportion that are inappropriate.

Design, setting and participants: The indications for ABG analysis were surveyed at a 58-bed level III ICU during fortnightly periods before and after a multifaceted educational intervention which included the introduction of a clinical guideline. The number of ABG tests performed during the period July-December 2017 was compared with that for the period July-December 2018. Tests were predefined as inappropriate if performed at regular time intervals, at change of shift, concurrently with other blood tests or after a treatment was ceased on a stable patient or after ventilatory support or oxygen delivery was decreased in an otherwise stable patient. The study was enrolled on the Quality Improvement Projects Register and ethics approval was waived by the local ethics committee.
\end{abstract}

Results: There was a $31.3 \%$ bed-day adjusted decrease in number of $A B G$ tests performed (33 $005 \vee 22$ 408; $P<0.001)$, representing an annual saving of $A \$ 770000$ and 100 litres of blood. The proportion of inappropriate ABG tests decreased by $47.3 \%(54.2 \% \vee 28.6 \% ; P<0.001)$ and the number of inappropriate $A B G$ tests per bed-day decreased by $71 \%(2.8 \vee 0.8 ; P<0.001)$. Patient outcomes before and after the intervention did not differ (standardised mortality ratio, $0.65 \vee 0.63 ; P=0.22$ ).

Conclusion: Staff education and implementation of a clinical guideline resulted in substantial decreases in the number of $A B G$ tests performed and the proportion of inappropriate $A B G$ tests.

Crit Care Resusc 2020; 22 (4): 370-377 


\section{ORIGINAL ARTICLES}

determine whether the number of unnecessary $A B G$ tests can be reduced without compromising patient care after introducing local clinical guidelines and staff education. We hypothesised that the proportion of unnecessary ABG tests, and the total number performed per bed-day, could be decreased by at least $25 \%$ without demonstrable negative effects on patient outcomes.

\section{Methods}

\section{Setting}

The study was conducted in the ICU of Royal North Shore Hospital in Sydney, Australia. This university-affiliated 58-bed level III ICU includes four "pods" — two 16-bed general ICUs, a 13-bed cardiothoracic ICU and a 13-bed neurosciences ICU. Royal North Shore Hospital has about 3700 ICU admissions each year across all major medical and surgical subspecialties, including trauma, spinal cord injuries and severe burns.

\section{Study design}

The processing fee and cost of consumables per ABG test were ascertained as follows. In Australia, the Medicare Benefits Schedule fee for $A B G$ analysis is $A \$ 33.70$ per test. At our institution, the cost is $A \$ 37.25$, of which $A \$ 35.34$ is the fee charged to the ICU department by the pathology service and $A \$ 1.91$ is the cost of consumables such as syringes and gauze. Volume of blood used was estimated from the recommended discard volume $(3-5 \mathrm{~mL})$ and sample volume $(0.5-1 \mathrm{~mL})$ in the ICU and local health district blood sampling guidelines. Time spent per test was estimated from local observation and published data. ${ }^{15,19}$ The total number of $A B G$ tests performed in each of the four pods during the period July-December 2017 was recorded. Subsequently, a multifaceted educational intervention was developed and implemented during the period January-June 2018. Following this, the number of $A B G$ tests during the period July-December 2018 was recorded. Data on occupancy, demographics, illness severity and patient outcomes for the periods before and after the intervention were compared.

\section{Figure 1. Decision flow chart included in the clinical guideline for arterial blood gas (ABG) testing}
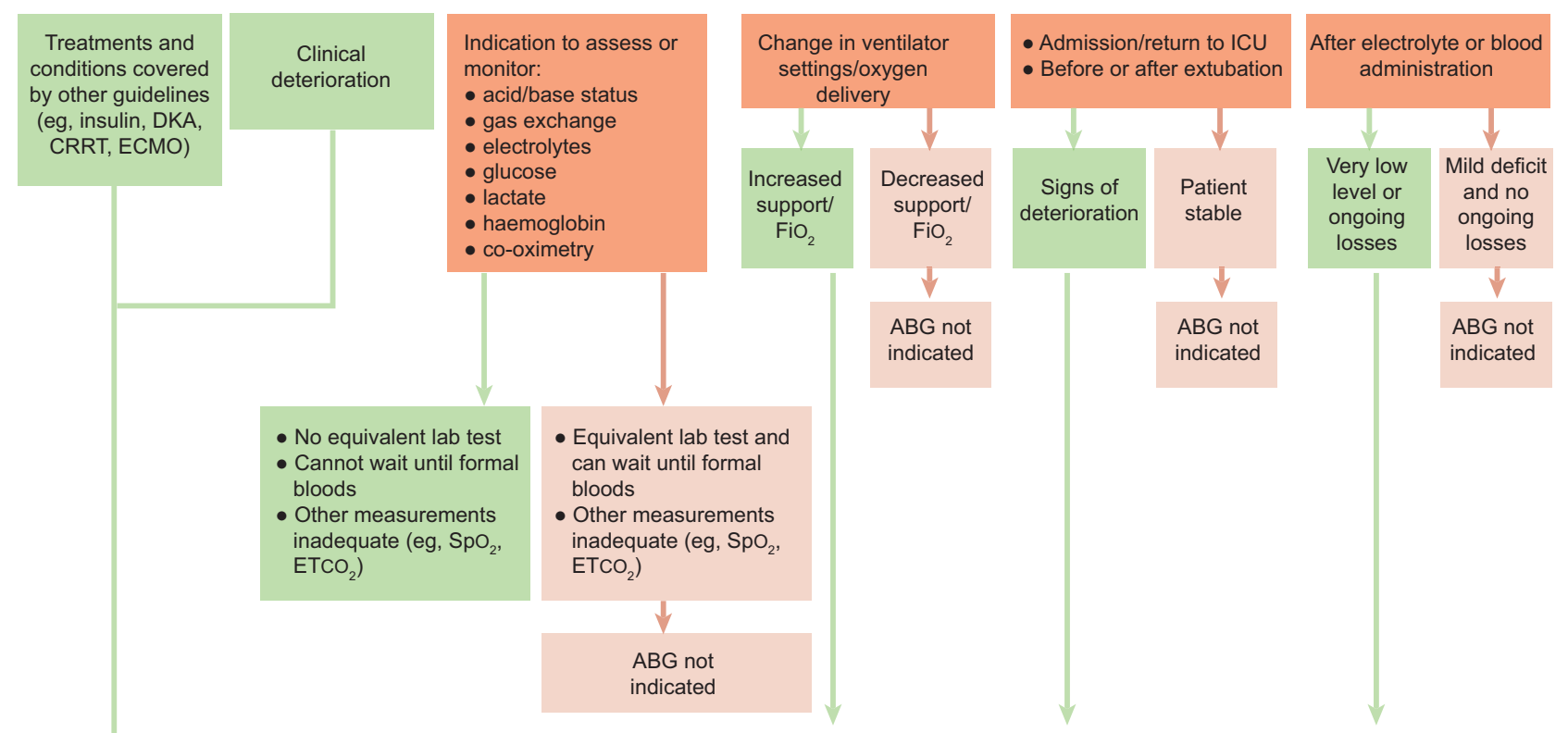

ABG may be indicated

$\mathrm{CRRT}=$ continuous renal replacement therapy; $\mathrm{DKA}=$ diabetic ketoacidosis; $\mathrm{ECMO}=$ extracorporeal membrane oxygenation; $\mathrm{ETCO}_{2}=\mathrm{end}^{-}$ tidal carbon dioxide; $\mathrm{FiO}_{2}=$ fraction of inspired oxygen; $\mathrm{ICU}=$ intensive care unit; $\mathrm{SpO}_{2}=$ pulse oximetry. 


\section{ORIGINAL ARTICLES}

The intervention focused on exploring appropriate and inappropriate indications for testing, followed by education of clinical staff with respect to the number of $A B G$ tests performed, and the associated financial, blood loss and labour costs. Nursing staff were educated via case-based in-service presentations, delivered by a nurse educator to small groups of clinical nurses twice a week in each pod for 12 weeks. Two presentations were made to medical and senior nursing staff at the departmental meeting. Finally, development of a local guideline for $A B G$ testing, including an easy-to-follow decision flowchart (Figure 1), reaffirmed the importance of clinically appropriate use of $A B G$ tests. In particular, it was emphasised that $A B G$ tests were not warranted at scheduled time intervals, in response to reduced ventilatory support, or routinely before or after extubation. ${ }^{25-27}$ It was also emphasised that laboratory tests should be performed in preference to point-of-care tests for parameters that can be measured by both methods (eg, haemoglobin), unless an urgent result was required. There was a de-emphasis on sampling after potassium replacement or blood transfusion, except in cases of severe hypokalaemia or anaemia, or in the setting of significant ongoing losses of potassium or bleeding. Clinicians were encouraged to monitor parameters clinically where possible; for example, using pulse oximetry $\left(\mathrm{SpO}_{2}\right)$ and end-tidal carbon dioxide $\left(\mathrm{ETCO}_{2}\right)$ as surrogates for arterial oxygen $\left(\mathrm{PaO}_{2}\right)$ and carbon dioxide $\left(\mathrm{PaCO}_{2}\right)$ tension in stable patients. Finally, educational posters were displayed in the $I C U$, and educational snippets were included in the local ICU newsletter and on closed social media groups.

To determine the indications for $A B G$ tests, staff were surveyed during two randomly selected 2-week periods, before and after the intervention. They were asked to complete a survey alongside all $A B G$ tests to ascertain their indication and whether they were initiated by medical or nursing staff. Indications were predefined as inappropriate if performed at regular time intervals, at change of shift, concurrently with other blood tests or after a treatment was ceased on a stable patient, or after ventilatory support or oxygen delivery was decreased in an otherwise stable patient All other indications were considered appropriate. Responses from the surveys were assumed to be representative of the entire respective 6 -month study period and were then extrapolated to overall $A B G$ use.

\section{Outcomes}

The primary outcome of our study was the total number of $A B G$ tests performed per bed-day. Secondary outcomes were the proportion of total $A B G$ tests that were inappropriate and the total number of inappropriate $A B G$

Figure 2. Number of $A B G$ tests per bed-day, by month, before and after the intervention

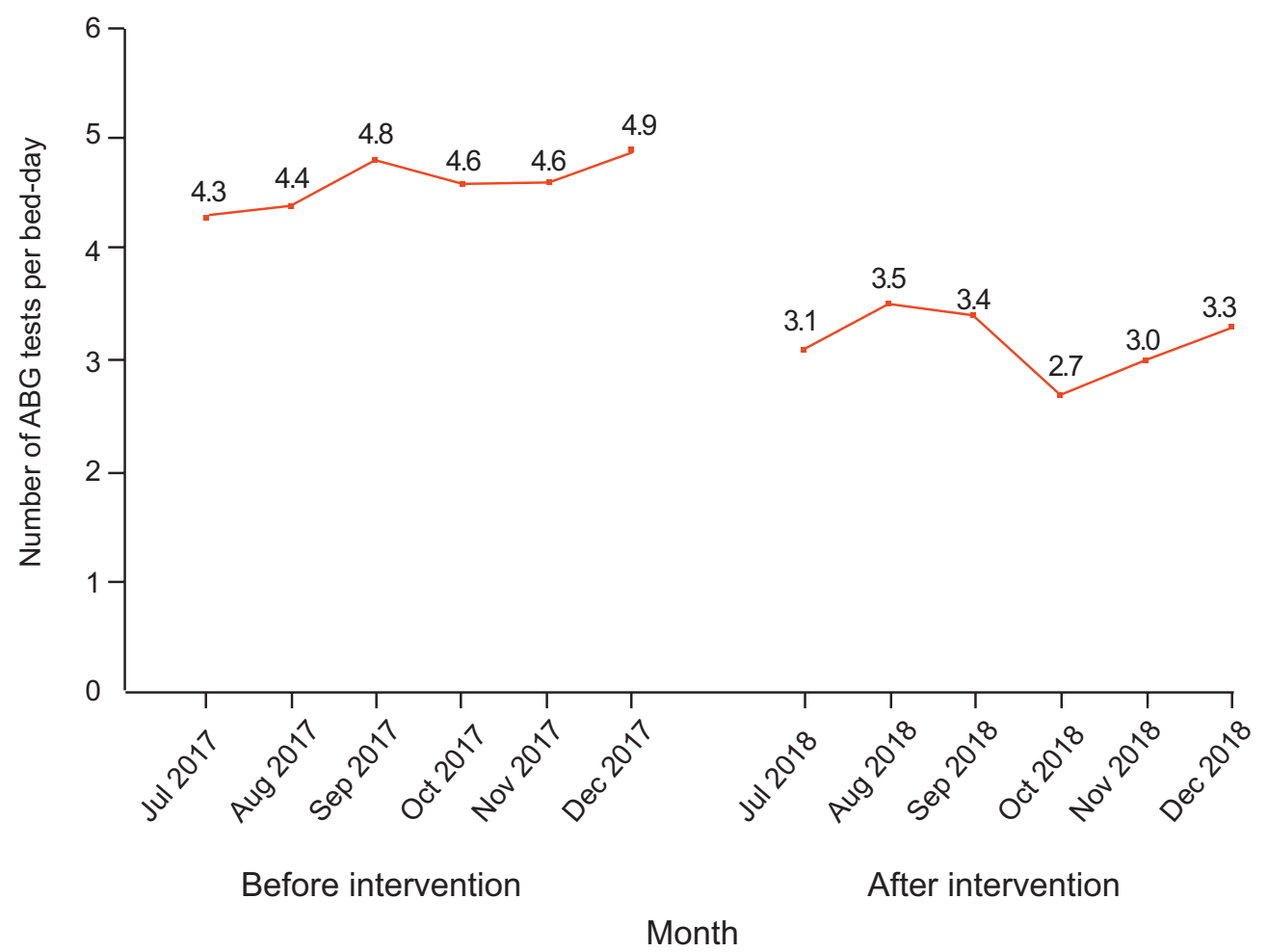


Figure 3. Number of arterial blood gas (ABG) tests performed and number of bed-days for general intensive care unit 1, by month, before and after the intervention

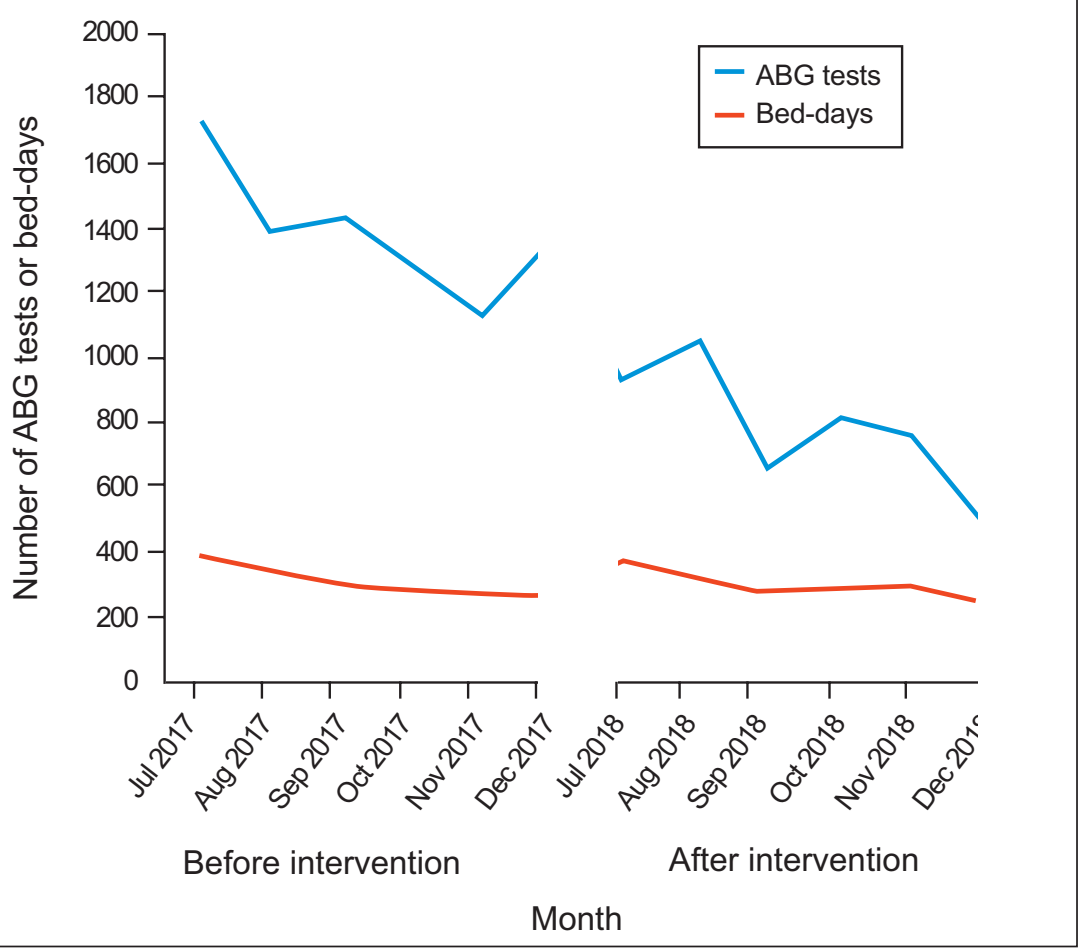

Figure 4. Number of arterial blood gas (ABG) tests performed and number of bed-days for general intensive care unit 2, by month, before and after the intervention

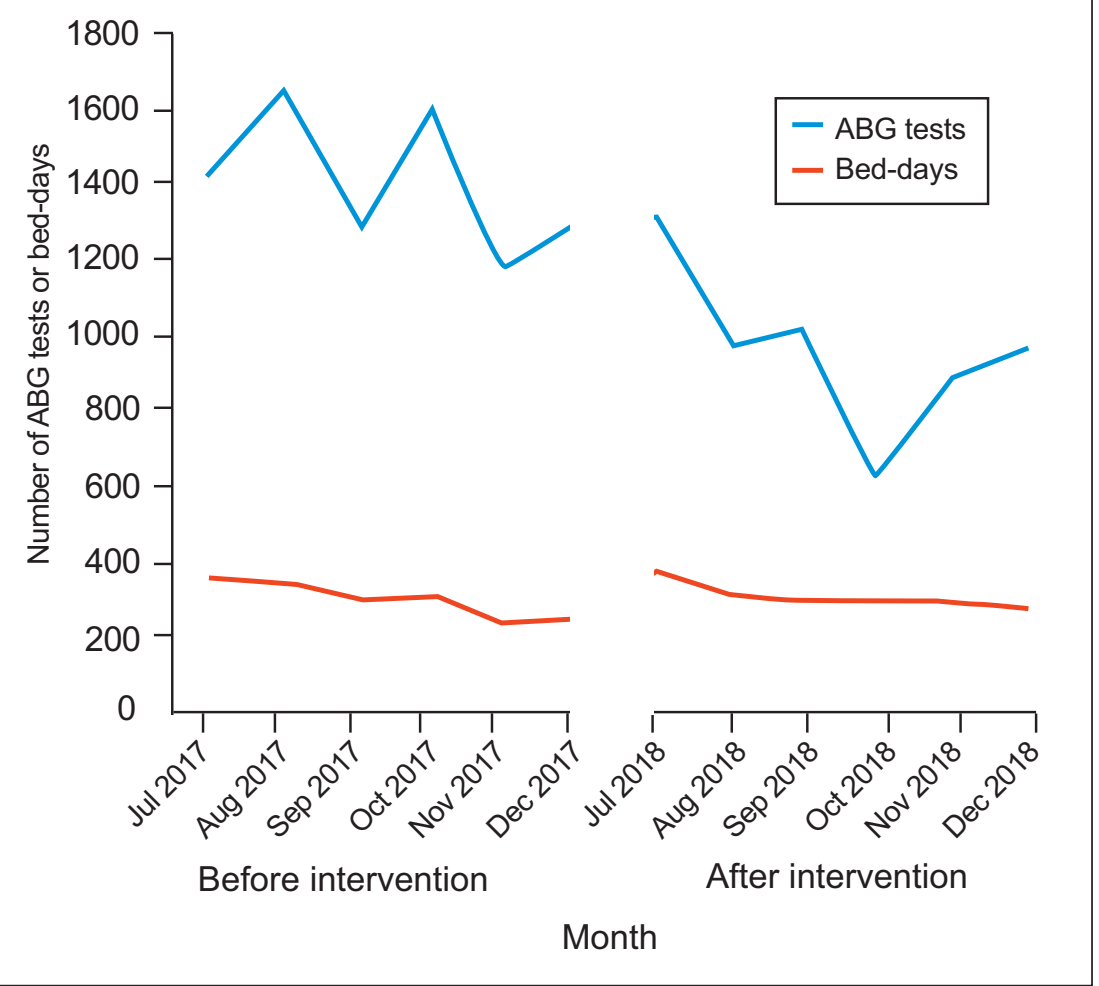

tests per bed-day. Data are presented as mean \pm standard deviation and median (interquartile range) for normal and non-normal continuous data, respectively, and as number (\%) for categorical data. Mann-Whitney $U$ tests, $t$ tests and $\chi^{2}$ tests were used as appropriate. Multivariable regression was performed to determine the effect of study period on ICU mortality and ICU length of stay when adjusted for age, sex, urgency of admission and APACHE (Acute Physiology and Chronic Health Evaluation) III scores. $P$ values less than 0.05 were deemed significant. All statistical analyses were performed using $R$ version 3.4.2 (R Foundation for Statistical Computing, Vienna, Austria). The study was enrolled in the local Quality Improvement Projects Register and the need for ethics approval was waived by the local ethics committee.

\section{Results}

\section{Numbers of $A B G$ tests}

In the 6 months before the intervention, 33005 ABG tests were performed, compared with 22408 in the 6 months after - a reduction of $32.1 \%$. When adjusted for occupancy (7208 v 7135 bed-days), there was a $31.3 \%$ reduction from 4.6 to $3.1 \mathrm{ABG}$ tests per bedday $(P<0.001)$ (Figure 2$)$. Each of the four pods achieved a decrease of at least $20 \%$ in the number of $A B G$ tests performed per bed-day; the general ICUs demonstrated $32.2 \%$ and $39.9 \%$ reductions (Figure 3 and Figure 4), the neurosciences ICU 34.4\% (Figure 5) and the cardiothoracic ICU 20.1\% (Figure 6).

\section{Numbers of inappropriate ABG tests}

After implementation of the clinical guideline, the proportion of inappropriate ABG tests fell by $47.3 \%$ (absolute proportions, $54.8 \%$ v $28.9 \%$; $P<0.001)$, as shown in Table 1 . When adjusted for occupancy (570 v 528 beddays) and for the number of $A B G$ tests performed (2869 $\vee$ 1397) before and 
Figure 5. Number of arterial blood gas (ABG) tests performed and number of bed-days for the neurosciences intensive care unit, by month, before and after the intervention

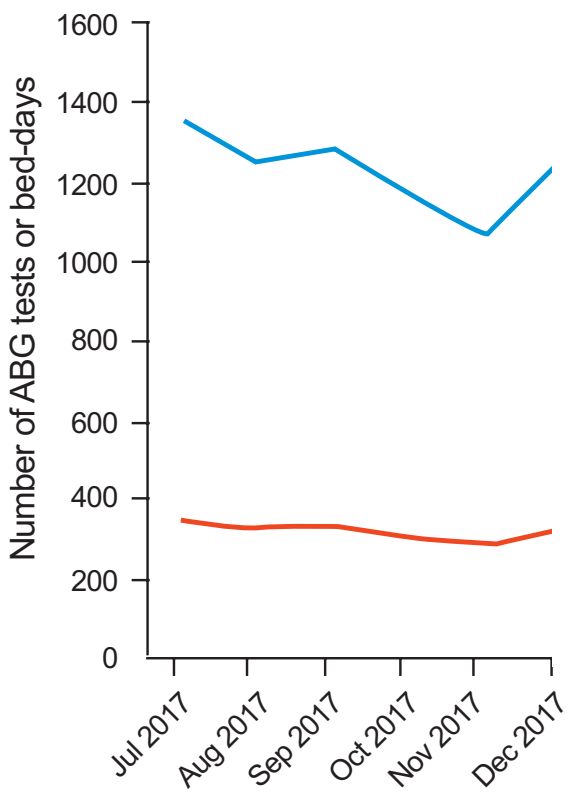

Before intervention
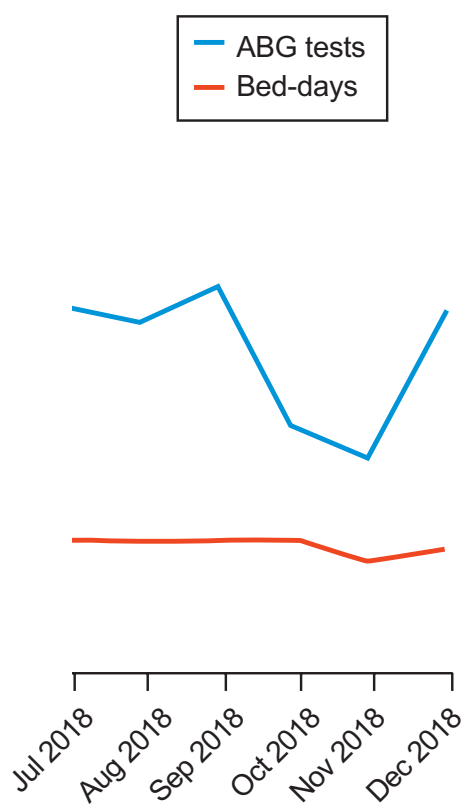

After intervention
Month

Figure 6. Number of arterial blood gas (ABG) tests performed and number of bed-days for the cardiothoracic intensive care unit, by month, before and after the intervention

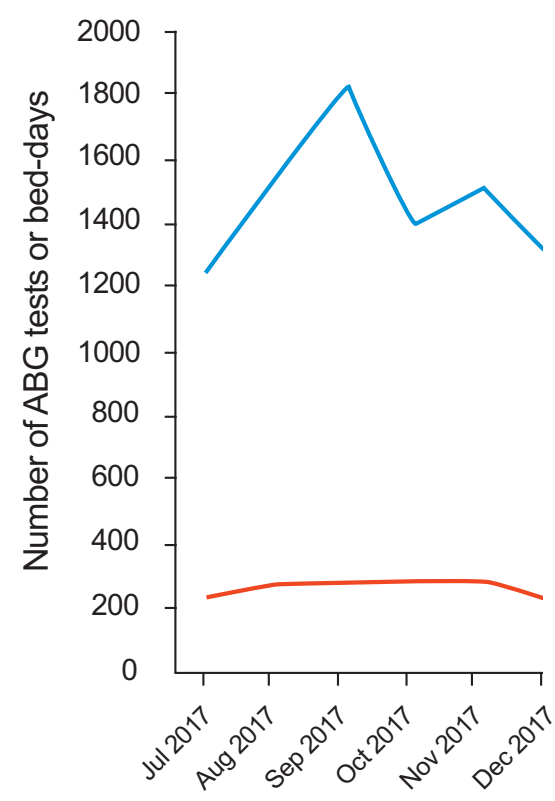

Before intervention
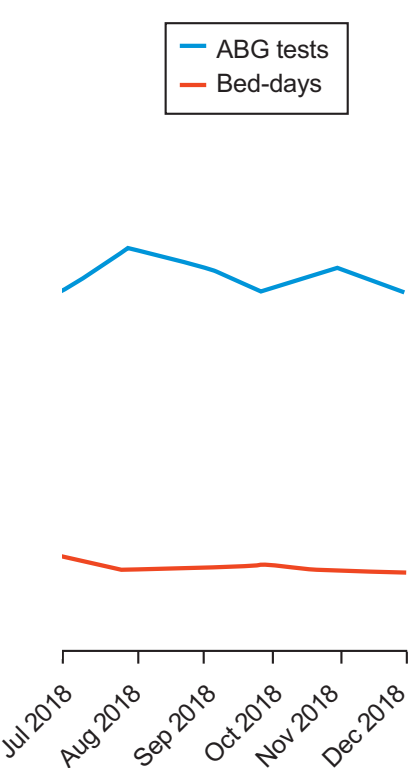

After intervention
Month after the intervention, there was a $71 \%$ absolute reduction in the number of inappropriate $A B G$ tests performed per bed-day $(2.8 \vee 0.8 ; P<0.001)$.

\section{Clinician-initiated tests}

The number of doctor-initiated $A B G$ tests per bed-day was 0.48 before the intervention and 0.58 after the intervention, corresponding to a proportion of doctor-initiated tests increasing from $10 \%$ to $22 \%$. The number of nurseinitiated $A B G$ tests per bed-day decreased from 4.4 to 2.0 , corresponding to a change from $88 \%$ to $77 \%$ of all tests. Fewer than $2 \%$ of all survey responses did not specify the clinician initiating the test.

\section{Cost implications}

Based on our calculation of $A \$ 37.25$ per sample, the estimated annual cost of $A B G$ tests before the intervention was $A \$ 2.45$ million. These samples involved the use of 330 litres of patient blood and 5500 hours of labour (about three full-time equivalent nursing staff). A $31.3 \%$ reduction in the number of $A B G$ tests represents annual savings of $A \$ 770$ 000, more than 100 litres of blood and one full-time equivalent member of staff in labour costs.

\section{Patient outcomes}

No significant differences in patient demographics, severity of illness, urgency of admission, period of mechanical ventilation or mortality rates were seen between the two study periods (Table 2). Multivariable regression did not identify any significant differences in ICU mortality when adjusted for age, sex, urgency of admission and APACHE III score (odds ratio, $1.001 ; 95 \% \mathrm{Cl}, 0.726-1.380 ; P=0.995)$. Similarly, there was no difference in ICU length of stay when adjusted for the same factors (odds ratio, 1.069; $95 \% \mathrm{Cl}$, $0.754-1.513 ; P=0.709$ )

\section{Discussion}

\section{Summary of findings}

Following our intervention, the annual expected number of inappropriate $A B G$ 


\section{ORIGINAL ARTICLES}

\begin{tabular}{|c|c|c|c|c|}
\hline \multirow[b]{2}{*}{ Reason and subcategory } & \multicolumn{2}{|c|}{ Before intervention } & \multicolumn{2}{|c|}{ After intervention } \\
\hline & $\begin{array}{c}\text { Number }(\%) \\
\text { ABG tests } \\
(n=709)\end{array}$ & Per bed-day & $\begin{array}{c}\text { Number }(\%) \\
\text { ABG tests } \\
(n=417)\end{array}$ & Per bed-day \\
\hline Deterioration & $57(8.0 \%)$ & 0.40 & $74(17.7 \%)$ & 0.47 \\
\hline Guide current treatment & $275(38.8 \%)$ & 1.95 & $210(50.4 \%)$ & 1.33 \\
\hline Change infusion & $120(16.9 \%)$ & 0.85 & $127(30.5 \%)$ & 0.81 \\
\hline Change oxygen delivery* & $65(9.2 \%)$ & 0.46 & $18(4.3 \%)$ & 0.11 \\
\hline Change ventilator settings* & $57(8.0 \%)$ & 0.40 & $18(4.3 \%)$ & 0.11 \\
\hline Other & $24(3.4 \%)$ & 0.17 & $38(9.1 \%)$ & 0.24 \\
\hline None selected & $9(1.3 \%)$ & 0.07 & $9(2.2 \%)$ & 0.06 \\
\hline After treatment ceased & $76(10.7 \%)$ & 0.54 & $44(10.6 \%)$ & 0.28 \\
\hline After extubation* & $21(3.0 \%)$ & 0.15 & $3(0.7 \%)$ & 0.02 \\
\hline $\begin{array}{l}\text { After electrolyte replace- } \\
\text { ment* }\end{array}$ & $38(5.4 \%)$ & 0.27 & $25(6.0 \%)$ & 0.16 \\
\hline After blood transfusion* & $12(1.7 \%)$ & 0.09 & $15(3.6 \%)$ & 0.10 \\
\hline Other* & $2(0.3 \%)$ & 0.02 & $1(0.2 \%)$ & 0.01 \\
\hline None selected* & $3(0.4 \%)$ & 0.02 & 0 & 0 \\
\hline Other & $298(42.0 \%)$ & 2.11 & $85(20.4 \%)$ & 0.54 \\
\hline Abnormal previous results & $67(9.4 \%)$ & 0.47 & $27(6.5 \%)$ & 0.17 \\
\hline With formal blood tests* & $52(7.3 \%)$ & 0.37 & $9(2.2 \%)$ & 0.06 \\
\hline Start/change shift* & $66(9.3 \%)$ & 0.47 & $8(2.0 \%)$ & 0.05 \\
\hline Not done for __hours* & $73(10.3 \%)$ & 0.52 & $23(5.5 \%)$ & 0.15 \\
\hline Prepare for transport & $5(0.7 \%)$ & 0.04 & $1(0.2 \%)$ & 0.01 \\
\hline Other & $33(4.7 \%)$ & 0.24 & $17(4.1 \%)$ & 0.11 \\
\hline None selected & $2(0.3 \%)$ & 0.02 & 0 & 0 \\
\hline None selected & $3(0.4 \%)$ & 0.02 & $4(1.0 \%)$ & 0.03 \\
\hline Total & $709(100 \%)$ & 5.02 & $417(100 \%)$ & 2.65 \\
\hline
\end{tabular}

$\mathrm{ABG}=$ arterial blood gas. ${ }^{*}$ Reasons deemed inappropriate.

tests declined from 40180 to 11565 . This type of reduction has benefits to hospital finances and resource allocation and is likely to be beneficial to patients. We observed a marked decline in nurse-initiated tests and a corresponding rise in doctor-initiated tests, which was likely achieved by empowering nurses to apply their clinical judgement to ABG testing, with the aid of a guideline and algorithm. This effect was sustained throughout the same period in the following year (July-December 2019), during which there were 3.1 ABG tests performed per bed-day (23 212 ABG tests and 7442 bed-days) (unpublished data).

\section{Comparison to other studies}

These results support findings from previous studies (many of which are several decades old) showing that simple educational interventions, including the development of a clinical guideline, can significantly decrease both the proportion of inappropriate $A B G$ tests and the total number of $A B G$ tests performed in ICUs. More than $50 \%$ of our pre-intervention $A B G$ tests were inappropriate, while other studies have found that $33-66 \%$ of all $A B G$ tests were unnecessary. Following our education program, we identified a $47 \%$ relative reduction in the proportion of inappropriate $A B G$ tests, which is consistent with $28-57 \%$ reductions seen in other studies. ${ }^{14,15,17-20}$ Interestingly, no previous studies have specified an absolute reduction in the number of inappropriate $A B G$ tests per bed-day, which we believe is a useful indicator given the large decrease in the denominator (total number of $A B G$ tests) after the intervention. Similar to findings of other small studies in North America and Europe, this initial Australian study demonstrated no detectable negative impacts on patient outcomes.

\section{Strengths and weaknesses}

A limitation of this study is that it was observational in nature and conducted over two periods. The same period of the year was analysed before and after the intervention, to avoid seasonally related variation in results. During the study there were no easily identifiable confounders, such as major changes in staffing or protocols other than introduction of the ABG clinical guideline. The study was a single-centre and non-blinded trial, but the intervention was easily achievable, inexpensive, and provided encouraging results across a heterogenous patient cohort. The cardiothoracic ICU predictably had the smallest reduction in ABG tests performed per bed-day owing to existing protocols that govern patients who have undergone cardiac surgery, which mandate regular $A B G$ tests in the first 48 hours after 


\section{ORIGINAL ARTICLES}

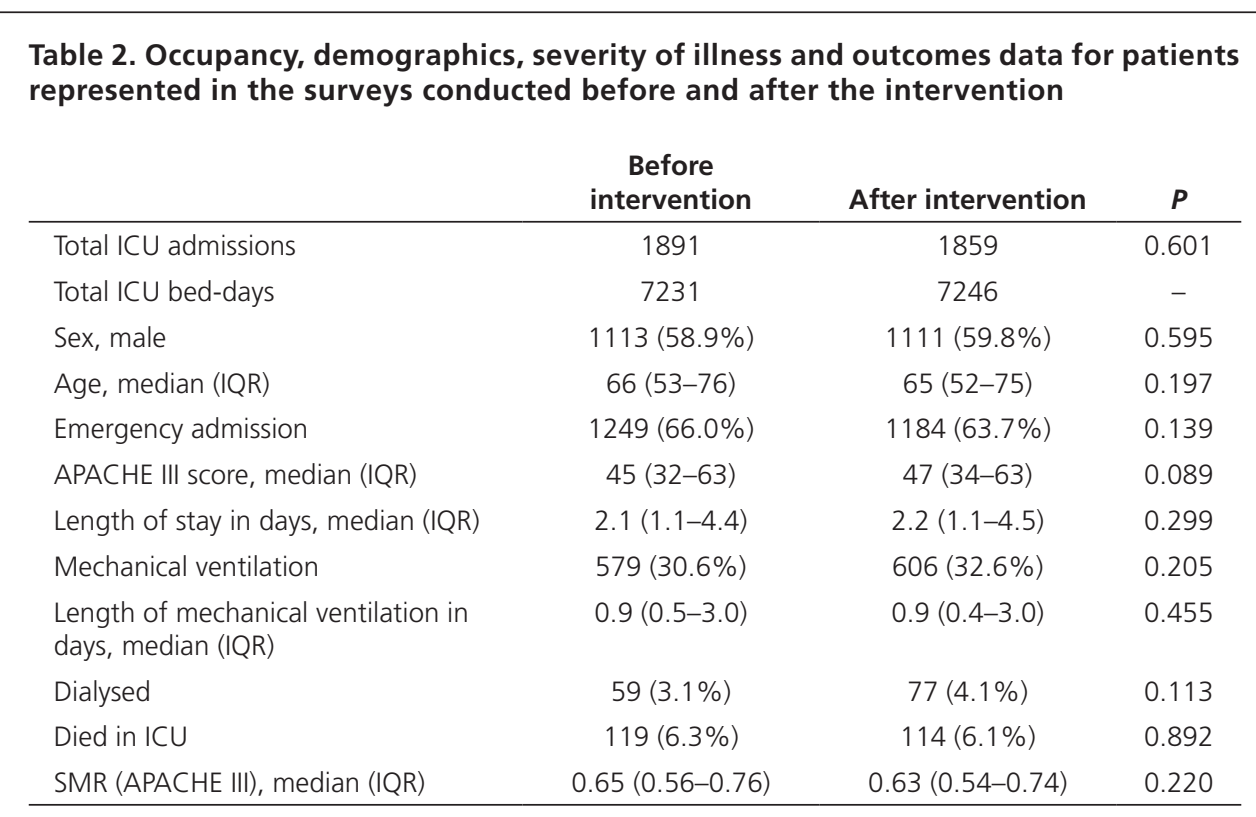

APACHE = Acute Physiology and Chronic Health Evaluation; ICU = intensive care unit; $\mathrm{IQR}=$ interquartile range; $\mathrm{SMR}=$ standardised mortality ratio.

the two countries, but if a similar proportional $31.3 \%$ reduction in $A B G$ tests could be achieved across all ICUs in Australia and New Zealand, savings could be made of up to $A \$ 33$ million, 4400 litres of blood and 40 full-time equivalent staff ( 73000 hours of labour). Furthermore, this framework could be applied to other critical care areas such as coronary care units and to rationalising ordering of other common tests in ICU, such as laboratory blood tests.

\section{Conclusion}

In conclusion, education of staff and introduction of a clinical guideline significantly reduced the number of $A B G$

surgery. To assess the economic impact of our intervention, we performed a simple cost-minimisation analysis, ${ }^{28}$ making the assumption that the clinical effect of decreasing the number of inappropriate $A B G$ tests would be minimal. Our outcome data suggest that this type of analysis was appropriate, although future investigators could consider performing a more formal cost-benefit health economic evaluation, including the collection of more granular data such as volume of blood sampled, time taken to perform tests and the need for blood transfusion.

\section{Local implications}

Titration of insulin infusions accounted for $22 \%$ of the total number of $A B G$ tests in the period after the intervention. About $10 \%$ of our ICU patients are receiving an insulin infusion at any given time, and local policy mandates second-hourly $A B G$ tests for these patients owing to concerns regarding the reliability of bedside glucometers in critically ill patients. ${ }^{29}$ This alone would account for 15000 20000 samples annually, or about $40 \%$ of our ABG tests in the period after the intervention. When combined with other protocol-mandated ABG tests, such as for patients on continuous renal replacement therapy and those with diabetic ketoacidosis, the potential for further reductions in the number of tests at our institution is limited.

\section{Wider relevance}

Our 58 ICU beds represent $2.4 \%$ of all ICU beds in Australia and New Zealand. ${ }^{30}$ We recognise that there is variety in patient acuity, clinical practice and health care costs across tests performed and the proportion and overall number of inappropriate ABG tests. This effect was sustained on reanalysis 12 months after the initial intervention.

Acknowledgements: This project was initiated as part of the Appropriate Blood Tests project being undertaken by the Intensive Care New South Wales Clinical Best Practice Working Group, Agency for Clinical Innovation.

\section{Competing interests}

None declared.

\section{Author details}

Oliver M Walsh ${ }^{1,2}$

Katelyn Davis ${ }^{3}$

Jonathan Gatward ${ }^{3,4}$

1 The Canberra Hospital, Canberra, ACT, Australia.

2 Australian National University, Canberra, ACT, Australia.

3 Royal North Shore Hospital, Sydney, NSW, Australia.

4 University of Sydney, Sydney, NSW, Australia.

Correspondence: oliver.mark.walsh@gmail.com

\section{References}

1 Critical Care Societies Collaborative - Critical Care. Five things physicians and patients should question. 2014. http:// 


\section{ORIGINAL ARTICLES}

www.choosingwisely.org/societies/critical-care-societiescollaborative-critical-care (viewed Oct 2020).

2 College of Intensive Care Medicine of Australia and New Zealand. Choosing Wisely. https://www.cicm.org.au/ Resources/Choosing-Wisely (viewed Oct 2020).

3 Angus DC, Deutschman CS, Hall JB, et al. Choosing wisely in critical care: maximizing value in the intensive care unit. Crit Care Med 2014; 42: 2437-8.

4 Rodriguez RM, Corwin HL, Gettinger A, et al. Nutritional deficiencies and blunted erythropoietin response as causes of the anemia of critical illness. J Crit Care 2001; 16: 36-41.

5 Corwin HL, Gettinger A, Pearl RG, et al. The CRIT study: anemia and blood transfusion in the critically ill - current clinical practice in the United States. Crit Care Med 2004; 32: 39-52.

6 Vincent JL, Baron JF, Reinhart K, et al. Anemia and blood transfusion in critically ill patients. JAMA 2002; 288: 1499507.

7 Barie PS. Phlebotomy in the intensive care unit: strategies for blood conservation. Crit Care 2004; 8 Suppl 2: S34-6.

8 Corwin HL, Parsonnet KC, Gettinger A. RBC transfusion in the ICU. Is there a reason? Chest 1995; 108: 767-71.

9 Hayden SJ, Albert TJ, Watkins TR, Swenson ER. Anemia in critical illness: insights into etiology, consequences, and management. Am J Respir Crit Care Med 2012; 185: 1049-57.

10 Astles T. Iatrogenic anaemia in the critically ill: a survey of the frequency of blood testing in a teaching hospital intensive care unit. J Intensive Care Soc 2009; 10: 279-81.

11 Ullman AJ, Keogh S, Coyer F, et al. 'True Blood' The Critical Care Story: an audit of blood sampling practice across three adult, paediatric and neonatal intensive care settings. Aust Crit Care 2016; 29: 90-5.

12 Andrews T, Waterman H, Hillier V. Blood gas analysis: a study of blood loss in intensive care. J Adv Nurs 1999; 30: 851-7.

13 Blum FE, Lund ET, Hall HA, et al. Reevaluation of the utilization of arterial blood gas analysis in the intensive care unit: effects on patient safety and patient outcome. J Crit Care 2015; 30: 438.e1-5.

14 Browning J, Kaiser D, Durbin C. The effect of guidelines on the appropriate use of arterial blood gas analysis in the intensive care unit. Respir Care 1989; 34: 269-76.

15 Merlani P, Garnerin P, Diby M, et al. Linking guideline to regular feedback to increase appropriate requests for clinical tests: blood gas analysis in intensive care. BMJ 2001; 323: 620-4.

16 Muakkassa FF, Rutledge R, Fakhry SM, et al. ABGs and arterial lines: the relationship to unnecessarily drawn arterial blood gas samples. J Trauma 1990; 30: 1087-93; discussion 93-5.

17 Pilon CS, Leathley M, London R, et al. Practice guideline for arterial blood gas measurement in the intensive care unit decreases numbers and increases appropriateness of tests. Crit Care Med 1997; 25: 1308-13.

18 Roberts D, Ostryzniuk P, Loewen E, et al. Control of blood gas measurements in intensive-care units. Lancet 1991; 337: 1580-2.

19 Martinez-Balzano CD, Oliveira P, O'Rourke M, et al. An educational intervention optimizes the use of arterial blood gas determinations across ICUs from different specialties: a quality-improvement study. Chest 2017; 151: 579-85.

20 Melanson SE, Szymanski T, Rogers SO, et al. Utilization of arterial blood gas measurements in a large tertiary care hospital. Am J Clin Pathol 2007; 127: 604-9.

21 Wang TJ, Mort EA, Nordberg P, et al. A utilization management intervention to reduce unnecessary testing in the coronary care unit. Arch Intern Med 2002; 162: 1885-90.

22 Low LL, Harrington GR, Stoltzfus DP. The effect of arterial lines on blood-drawing practices and costs in intensive care units. Chest 1995; 108: 216-9.

23 Zimmerman JE, Seneff MG, Sun X, et al. Evaluating laboratory usage in the intensive care unit: patient and institutional characteristics that influence frequency of blood sampling. Crit Care Med 1997; 25: 737-48.

24 Diby M, Merlani P, Garnerin P, Ricou B. Harmonization of practice among different groups of caregivers: a guideline on arterial blood gas utilization. J Nurs Care Qual 2005; 20: 327-34.

25 Pawson SR, DePriest JL. Are blood gases necessary in mechanically ventilated patients who have successfully completed a spontaneous breathing trial? Respir Care 2004; 49: 1316-9.

26 Salam A, Smina M, Gada P, et al. The effect of arterial blood gas values on extubation decisions. Respir Care 2003; 48: 1033-7.

27 See KC, Phua J, Mukhopadhyay A. Monitoring of extubated patients: are routine arterial blood gas measurements useful and how long should patients be monitored in the intensive care unit? Anaesth Intensive Care 2010; 38: 96-101.

28 Cox HL, Laupland KB, Manns BJ. Economic evaluation in critical care medicine. J Crit Care 2006; 21: 117-24.

29 Pereira A, Cavalcanti A, Correa T, et al. Reliability of arterial, capillary and venous point-of-care glucose measurements in the intensive care unit setting: evaluation of two glucometers. Crit Care 2007; 11 Suppl 2: P140.

30 Australian and New Zealand Intensive Care Society. ANZICS Centre for Outcome and Resource Evaluation annual report 2017. Melbourne: ANZICS, 2017. 